\title{
Prolotherapy for early osteoarthritis knee
}

\author{
Jain $\mathbf{S}^{1}$, Jain $\mathbf{R}^{2}$, Arora $\mathbf{K K}^{3}$ \\ ${ }^{1}$ Dr Sarvesh Jain, Assistant Professor, Department of Anesthesia, Bundelkhand Medical College, Sagar. ${ }^{2}$ Dr Rajesh Jain \\ ,Assistant Professor, Department of Orthopedics, Bundelkhand Medical College, Sagar, ${ }^{3}$ Dr KK Arora, Professor, \\ Department Of Anesthesia, Bundelkhand Medical College, Sagar, India.
}

Address for correspondence: Dr Sarvesh Jain, Email: jainvidisha7@yahoo.co.in

\begin{abstract}
Introduction: Osteoarthritis is most prevalent arthritis in older patients. Present study is to evaluate efficacy of dextrose prolotherapy for treatment of early knee osteoarthritis. Material \& Method: This study was done at Bundelkhand Medical College, Sagar. In our study we studied 38 patients of more than 55 years of age. For treatment of Knee OA, 3 sitting of prolotherapy were given one month apart and patient followed up for 1 year. Results: $76 \%$ patients showed improvement on second follow-up. On third follow up $89 \%$ showed improvement. On further follow up at 6 months $81 \%$ of patients were symptomatically improved. These patients remained symptomatically better even at the conclusion of study. Discussion: Definitive mechanism of action of prolotherapy is unknown but probable mechanism is, hypertonic dextrose induced growth factor release and connective tissue healing thereafter. Conclusion: Dextrose prolotherapy injection is safe, reliable and long lasting treatment modality for early osteoarthritis
\end{abstract}

Keywords: Dextrose Prolotherapy, Knee Osteoarthritis, Prolotherapy.

\section{Introduction}

Osteoarthritis (OA) also known as degenerative arthritis or degenerative joint disease is a group of mechanical abnormalities involving degradation of joints. OA involves both degenerative and regenerative processes [1]. Pain may arise from joint capsule, ligaments, synovium, bone, the outer edge of the menisci as well as supportive extra-articular ligaments and tendons. Standard-of-care is multidisciplinary, often including physical therapy, anti-inflammatory medication, intra-articular viscosupplementation, and arthroscopic surgery. However, a recent systematic review reported no clear benefit of any one therapy. Other conservative therapies and oral supplements have also been reviewed. While some support exists for their use, definitive evidence is lacking. Acupuncture was reported as efficacious in a rigorous randomized controlled trial (RCT), though results were limited by substantial missing data and short follow-up period. In light of high prevalence and substantial impact on individuals and society, it is essential to search for its treatment in complimentary medicine.

Manuscript received: $22^{\text {nd }} J a n 2014$

Reviewed: $25^{\text {th }}$ Jan 2014

Author Corrected: $3^{\text {rd }}$ Feb 2014

Accepted for Publication: $7^{\text {th }}$ Feb 2014
Symptoms may include joint pain, tenderness, stiffness and locking. One of the features of OA is loss of cartilage. When bone surfaces become less well protected by cartilage, bone may be exposed and damaged. As a result of decreased movement secondary to pain, regional muscles may get atrophied and ligaments may become more lax. Knee is major weight bearing joint of body and it more prone to age related arthritis, it is equal in both sexes up to age of 55 years. After 55 it is more common in females. Treatment generally involves a combination of exercise, lifestyle modification, and analgesics. If pain becomes debilitating, joint replacement surgery may be used to improve the quality of life. Prolotherapy is also known as "proliferation therapy," "regenerative injection therapy "or proliferative injection therapy [2]. It is a new form of therapy, which is used in knee osteoarthritis.

A core principle is the injection of small volumes of an irritant solution at multiple painful ligament and tendon insertions and in adjacent joint spaces over several treatment sessions [3]. Contemporary hypotheses suggest that prolotherapy stimulates local healing of chronically injured extra- and intra-articular tissue, though definitive evidence is lacking. Prolotherapy works by raising growth factor levels or effectiveness in connective tissue ligaments and tendons to promote repair or growth [4]. Hypertonic dextrose is a commonly used injection. 
Prolotherapy injections target multiple potential pain generators in and around the knee joint; it may be wellsuited to address the multifactorial cause of knee pain from osteoarthritis.

\section{Material and Method}

After permission from ethical committee and written informed consent, study was conducted in Bundelkhand medical college, Sagar (MP) and sample were taken from general population with mixed socioeconomic status of Indian ethnicity We have studied 38 cases of early bilateral osteoarthritis knee, treated by prolotherapy at Bundelkhand medical college, sagar from 2009 to 2012.

We included only those patients who have early osteoarthritis (Radiological stage grade 1 and grade 2). Osteoarthritis based on clinical criteria (American College of Rheumatology), on physical examination, and moderate to severe knee pain for at least 3 months, (visual analogue score $>5$ ). All the patients are between the age group of 55 to 70

Out of 38 patients 28 were female and 10 were male. These patients initial treated by life style modifications and analgesics. Each was then observed for 1 year and evaluated on $13^{\text {th }}$ weeks, 6 months and on 1 year.

Exclusion criteria: We have excluded following patients from study.

1. Patients who had diabetes,

2. Patients on anticoagulation therapy.

3. History of total knee replacement.

4. Prior knee prolotherapy.

5. Any knee injection within 3 months.
6. Inflammatory or post infectious knee arthritis.

7. Daily use of opioid medication.

8. Allergy or intolerance to study medication and co morbidity severe enough to prevent participation in the study protocol and any other systemic illness.

We have given dextrose $15 \%$ solution made by diluting commercially available dextrose $25 \%$ solution with distilled water under aseptic condition after explaining procedure to the patient and taking written informed consent.

Injections were performed at 1,5 , and 9 weeks as OPD procedure. Total of $6 \mathrm{ml}$ of dextrose $15 \%$ given intraarticular and $1 \mathrm{ml}$ per tender site in anterior knee structure by palpation. Tender site injected were given at least 1 centimeter apart. Patients were sent home after 15 minutes of close watch for any allergic reaction and discharged on tablet paracetamol $650 \mathrm{mg}$ twice daily for 3 days after each treatment session. Patients were warned against possible worsening of pain for initial 2 -3 days. Patient was declared improved once his VAS [Visual analogue score] was reduced by 2 out of 10

\section{Results}

None of the patient showed any improvement on 5th week (first follow up). On 9th week (on second follow up) out of 38 patients 29 showed improvement. On 13th week ( 3 rd follow up) 34 showed improvement. After 6 months out 34, 31 patients remained improved; rest 3 patients showed deterioration and reached to pretreatment stage. On 1 year follow up all the 31 improved patients remained the same

Table: Follow up results

\begin{tabular}{|l|l|l|l|}
\hline Follow up & $\begin{array}{l}\text { Number of patients } \\
\text { showed improvement }\end{array}$ & $\begin{array}{l}\text { Number of patients } \\
\text { showed no improvement }\end{array}$ & $\begin{array}{l}\text { Number of patients } \\
\text { showed deterioration }\end{array}$ \\
\hline $1^{\text {st }}(5$ th week $)$ & nil & 38 & Nil \\
\hline $2^{\text {nd }}(9$ th week $)$ & 29 & 9 & Nil \\
\hline $3^{\text {rd }}(13$ th week $)$ & 34 & 4 & Nil \\
\hline $4^{\text {th }}(6$ month $)$ & 31 & 4 & 3 \\
\hline $5^{\text {th }}(1$ year $)$ & 31 & 4 & 3 \\
\hline
\end{tabular}

\section{Discussion}

A diagnosis of $\mathrm{OA}$ is mainly based on symptoms. A patient that has reached a certain age and has joint pain, limitation of movement, crepitus and, sometimes, effusion in the joint might get the diagnosis of OA. Recommendations for the diagnosis of knee OA include three main symptoms: knee pain, short-lived morning stiffness, and functional limitation in combination with three signs on physical examination (crepitus, restricted movement and bony enlargement). The treatment of OA is symptom driven (mainly pain and anti-inflammatory medication in combination with exercise treatment and lifestyle changes) but, unfortunately, such treatment 
cannot prevent or cure OA. Most of the diseasemodifying treatments were directed towards regeneration of the cartilage and were tested in patients with evident OA. However so far these efforts have not been very successful and have not had a significant influence on the symptoms of OA [5]. Injectable agents including intraarticular injections of Corticosteroid, hyaluronic acid, growth hormone, platelet-rich plasma and/or autologous blood injections all are used [6].

Prolotherapy is an injection therapy for chronic musculoskeletal injury, including knee osteoarthritis [4]. The mechanism of action for dextrose is unclear. Dextrose $15 \%$ has been hypothesized to stimulate healing of chronically injured extra- and intra-articular tissue. Animal model studies reported increased inflammatory markers in and around site of injection and significantly enlarged cross-sectional area in medial collateral ligaments. In addition to dextrose-specific effects, needle trauma and volume expansion of tissue may also produce tissue-level effects.

In our study we found significant improvement with. Even after 6 months $81 \%$ of patients remained improved. There after till the conclusion of study, improvement was remained same these findings suggest that dextrose prolotherapy may improve knee osteoarthritis for certain patients. This result is similar to the study by Jensen KT, et al who found that Prolotherapy resulted in clinically meaningful sustained improvement of pain, function, and stiffness scores for knee osteoarthritis compared with blinded saline injections and at-home exercises [7].

David Rabago et al studied Ninety adults with at least 3 months of painful knee osteoarthritis were randomized to blinded injection (dextrose prolotherapy or saline) or athome exercise found. No baseline differences existed between groups. All groups reported improved [8]

Reeves KD and Hassanein et al used Dextrose 10\% in one group and $.075 \%$ lignocaine in bactreriostatic water in another group. In their study, the magnitude of improvement in subjective and objective measures in control-treated knees suggested that the control solution (water) may have had more than a placebo effect. Nevertheless, Hotelling multivariate analysis of paired observations between 0 and 6 months for pain, swelling, buckling episodes, and flexion revealed significantly more benefit from the dextrose injection, $(p=.015)$. By 12 months (6 injections) the dextrose-treated knees improved in pain, swelling, knee buckling and flexion by $44 \%, 63 \%, 85 \%$ and 14 degrees respectively. Analysis of blinded radiographic readings of 0 and 12 month films revealed a statistically significant improvement in radiographic measures of osteoarthritis from 0 to 12 months [9].

Dumais Rand Benoit $\mathrm{C}$ et al used dextrose $15 \%$ at collateral ligaments and $20 \%$ inside the joint in one group and exercise alone in another group, their outcome measure was Western Ontario and McMaster Universities Osteoarthritis Index of severity of osteoarthrosis symptoms (WOMAC). They found dextrose prolotherapy group improved significantly [10]. Its use in clinical practice is relatively uncomplicated; prolotherapy is performed in the outpatient setting without ultrasound guidance using inexpensive solutions.

For responders, whether prolotherapy results in sustained effect past 52 weeks, disease modification, or delayed definitive care, such as knee replacement, is not known. Clinical experience suggests that repeated sessions and tune-up sessions after 52 weeks improve outcomes and do not pose additional risk.

Prolotherapy for knee osteoarthritis has not been compared with intra-articular corticosteroid and hyaluronic acid injections. Determination of clinical utility of prolotherapy will require confirmation in a larger effective trial. Prolotherapy performed by a trained operator resulted in safe, significant, and sustained improvements. Prolotherapy may be an appropriate therapy for patients with knee osteoarthritis refractory to conservative care. However larger size study is required to fully validate this theory

\section{Conclusion}

Prolotherapy is very effective in management of early stage of Osteoarthritis knee. Initial injection shows no improvement or worsening of symptoms. After 6 months of starting treatment patients once improved did not show deterioration, so this kind of therapy provided long term pain relief. It is relatively free from side effect. It does not require high degree of technical expertise, as knee injection can be done with basic skills and can be done in outpatient setting. Dextrose is relatively inexpensive.

Funding: Nil

Conflict of interest: Nil

Permission from IRB: Yes

\section{References}


1. Hashan manaletal.Clinical features and pathogenetic mechanisms of osteoarthritis of the hip and kneeI: BCMJ.2010; 52(8):393-398.

2 David Rabago, Jeffrey J. Patterson, Joel Jay Baumgartner. Prolotherapy. Jan 2011: 113-130

3. David Rabago, Jeffrey J.et al. Dextrose Prolotherapy for Knee Osteoarthritis: A Randomized Controlled Trial Ann Fam Med. 2013;11(3): 229-237.

4. Hackett GS, Hemwall GA,Montgomery GA Ligament and tendon relaxation treated by prolotherapy. 5th ed. Oak Park: Gustav A. Hemwall;1993.p.175

5. Kellgren JH, Jeffrey MR, Ball J The Epidemiology of Chronic Rheumatism. Atlas of Standard Radiographs of Arthritis. Oxford, UK:Blackwell Scientific Publications; 1963.p.198

6. Dong Rak Kwon and Gi Young Park.Intra-Articular Injections for the Treatment ofOsteoarthritis: Focus on the ClinicalUse of Several Regimens. Available at: http://www.researchgate.net/publication/221926910_Int ra-
Articular_Injections_for_the_Treatment_of_Osteoarthrit is_Focus_on_the_Clinical_Use_of_Several_Regimens. Cited on13th April 2014.

7.

Jensen KT, Rabago DP, Best TM, Patterson JJ, Vanderb y R Jr.Early inflammatory response of knee ligaments to prolotherapy in a rat model. J Ortho Res. 2008;26(6):816-823.

8. Rabago D, Patterson JJ, Mundt M, et al. Dextrose prolotherapy for knee osteoarthritis: a randomized controlled trial. Ann Fam Med. 2013;11:229-237.

9. Reeves KD Hassanein K. Randomized prospective double-blind placebo-controlled study of dextrose prolotherapy for knee osteoarthritis with or without ACL laxity. Alt Ther Hlth Med 2000;6(2):37-46.

10. Dumais R, Benoit C, Dumais A, Babin L, Bordage R, de Arcos C, Allard J, Bélanger M. Effect of Regenerative Injection Therapy on Function and Pain in Patients with Knee Osteoarthritis: A Randomized Crossover Study. Pain Med. 2012 Jul 3. doi: 10.1111/j.15264637.2012.01422.x.

\section{How to cite this article?}

Jain S, Jain R, Arora KK. Prolotherapy for early osteoarthritis knee. Int J Med Res Rev 2014;2(4):279- 282. doi: 10.17511/ijmrr.2014.i04.02 